\title{
La teología política de J. B. Metz y la teología de la liberación. Algunas consideraciones ${ }^{1}$
}

\author{
Juan Pablo Espinosa Arce \\ Profesor de religión y filosofía \\ (Universidad Católica del Maule)
}

Este artículo pretende dar cuenta de la relación existente entre la teología política del alemán Johann Baptist Metz y la teología latinoamericana de la liberación. En esa relación se puede percibir cómo el discurso sobre el Dios de Jesús de Nazaret, que nace en nuestro continente, influye positivamente en la propuesta teológica del teólogo alemán. Diversos encuentros de Metz con los teólogos latinoamericanos en la década de 1980, menciones explícitas de la teología de la liberación en buena parte de su obra y su prólogo a la edición alemana de Teología de la liberación. Perspectivas, de Gustavo Gutiérrez, indican que Metz recibió y valoró positivamente la creatividad latinoamericana.

\section{La teología política en el contexto del Vaticano II}

La teología política de Metz se sitúa dentro de la gran renovación teológica posterior al concilio Vaticano II (1962-1965). La década de 1960 es un compleja, per creativa, tanto en el campo de la historia social y cultural como en el de la historia de la Iglesia. Las intuiciones de Juan XXIII de aggiornar, de renovar la Iglesia y de abrir la ventana para dejar entrar aire fresco se manifestaron en una teología y en una praxis pastoral de cara al mundo, en diálogo con la cultura y sus manifestaciones. Junto con ello, se reconocieron los aspectos positivos de

1. Este artículo está basado en la tesis del autor, "Aportes de la teología política de Johann Baptist Metz a la comprensión de la eclesialidad de la fe", presentada para optar al grado de Magíster en Teología, en la Facultad de Teología de la Pontificia Universidad Católica de Chile. 
la modernidad y se llevó a cabo un discernimiento de los signos de los tiempos para escuchar la voz y los silencios de Dios en el presente. A juicio de J. Espeja (2000), dos teologías fueron significativas en la orientación del Vaticano II, a saber, la teología latinoamericana de la liberación y la teología política europea. Ambas teologías están emparentadas en su orientación social y política y en la importancia de la praxis, entendida como primer momento. La teología es el momento segundo, que busca comprender la acción concreta de los creyentes en el mundo.

La teología política buscó fundamentalmente dos cosas. En primer lugar, sugerir una nueva visión de la relación de la Iglesia con el mundo, basada en una teología del mundo. A propósito de esto, Metz había afirmado que "todo concilio supone un nuevo punto de partida y le asigna a la teología nuevas tareas. Una de estas tareas es la de recrear la relación entre Iglesia y mundo. Como teólogo fundamental, Metz intenta exponer la fe en una forma que esté en consonancia con la actual situación histórica"2. En otras palabras, Metz busca que su teología sea significativa para establecer puentes de diálogo con la cultura y la modernidad. El segundo elemento que busca la teología política, y que fundamenta nuestro artículo, es que "ante los terribles genocidios como el que tuvo lugar a mediados del siglo XX en Alemania, el interrogante parecía ineludible: ¿tiene algo que decir aquí la fe cristiana? La respuesta es afirmativa, pero a condición de que los creyentes no guarden silencio, sino que renueven y desarrollen crítica y conscientemente la dimensión política de su fe"3.

\section{Las tareas de la nueva teología política}

La reflexión teológica de Metz es una reacción a la crítica ilustrada francesa, conocida como "tarea negativa de la teología política". Sin embargo, es necesario reconocer que la crítica a la religión no es solo patrimonio de la Ilustración, sino que también se encuentra en otras manifestaciones filosóficas, sociológicas y antropológicas. Pese a ello, Metz se concentra en la Ilustración. La crítica de la Ilustración relegó la religión al ámbito privado. H. Zirker sostiene que la crítica ilustrada despoja a la Iglesia de las competencias sociales, originadas en la edad media. La Iglesia y el Estado se presentaban unidos, porque "todos los ámbitos de la vida tenían un carácter religioso tanto en su forma y expresión externa como en su contenido espiritual"4. La época moderna trajo un cambio profundo al centrarse en el hombre y dejar de lado el dato de Dios como algo insignificante para la maduración racional del ilustrado y de todo aquel que puede pensar por sí mismo. La religión replegada al ámbito privado y, por lo tanto, fundamentada

2. M. Ruz, Nueva teología política. Desarrollo del pensamiento de Johann Baptist Metz, p. 70 (Argentina: Universidad de Córdoba, 2010).

3. J. Espeja, Creer en este mundo, p. 70 (Madrid: BAC, 2000).

4. H. Zirker, Crítica a la religión, p. 41 (Madrid: Herder, 1985). 
en categorías ahistóricas y apolíticas, se caracteriza por no formar parte de la sociedad, ni proporcionar sentido al hombre.

La otra crítica dirigida contra la religión proviene del marxismo, que la entiende como "superestructura ideológica de una determinada praxis social y de determinadas relaciones de poder"5. Marx critica, en primer lugar, que la religión ya no expresa la conciencia comunitaria, sino que se ha convertido en medio para dividir, porque responde a los intereses de ciertos grupos de poder. En este contexto, formula la conocida expresión de que la religión es opio o adormecimiento del pueblo. De esa manera, la religión es relegada al ámbito de lo privado. Dado que la religión rehúye la conflictiva realidad histórica, Marx plantea volver a la realidad concreta para que el hombre se haga cargo de su presente.

Cuál es la reacción de la teología ante la crítica ilustrada y marxista. Según Gibellini, "la teología reaccionó retirándose a la esfera de lo privado [...] privatizó este mensaje en su núcleo esencial y redujo la praxis de la fe a la decisión amundana del individuo [...] las categorías dominantes en esta teología para la explicación del mensaje son prevalentemente categorías de lo íntimo, de lo privado, de lo apolítico". En definitiva, propuso vivir la fe como "una decisión de espaldas al mundo"7. Frente a la teología moderna, que considera al mundo como hostil y contrario al proyecto salvífico, Metz reacciona con la única teología que puede enfrentarse a la privatización de la fe, a saber, la teología política crítica, o la nueva teología política, porque es la que "toma en serio la dimensión social y política de la existencia humana tal y como se comprende hoy día"8. El proyecto de Metz es lograr que la "Teología contribuya a que la palabra de la fe vuelva a ser una palabra no solo privada, sino también socialmente eficaz"9.

Así, además de la crítica a la privatización excesiva y exagerada de la fe, producto de la crítica ilustrada y marxista, la teología política también tiene una dimensión positiva.

[D]esarrollar las implicaciones públicas y sociales del mensaje cristiano [...] se trata de responder críticamente a su desafío, asumiendo la tarea de desarrollar, también en teología, una nueva relación entre teoría y praxis. Y la teología puede hacerlo porque las promesas escatológicas de la tradición bíblica no son un horizonte vacío de espera religiosa, sino que tienen una

5. R. Gibellini, La teología del siglo XX, p. 322 (Santander: Sal Terrae, 1998).

6. Ibidem.

7. J. B. Metz, "El problema de una teología política", Concilium 36 (1968), 385-403, 387.

8. C. Geffré, "Historia reciente de la teología fundamental. Intento de interpretación", Concilium 46 (1969), 337-358, 356.

9. J. Illanes y J. Saranyana, Historia de la teología, p. 373 (Madrid: BAC, 1995). 
dimensión pública que debe hacerse valer en su función crítica y liberadora en relación al proceso histórico social ${ }^{10}$.

La explicitación de esa tarea positiva de la teología política, a la cual Metz llama "segunda reflexión", permite comprender qué quiere decir cuando habla de política. Lejos de significar una determinada opción partidista, expresa la profunda e insoslayable dimensión "pública y social" del cristianismo, la cual había sido olvidada a causa de las críticas modernas. Esta dimensión pública se divide a su vez en una vertiente crítica y liberadora, con vistas a un proceso histórico y social. Por lo tanto, la nueva teología política hace referencia, constante y necesaria, a la historia y a los procesos sociales, en los cuales la fe se expresa como memoria, narración y solidaridad, conceptos fundamentales de dicha teología política. Esta función, en palabras de Metz, busca "determinar las relaciones existentes entre la religión y el cuerpo social, entre la Iglesia y la dimensión pública de la sociedad, entre la fe escatológica y la actividad social"11.

\section{Metz y la teología de la liberación}

A propósito del contacto de Metz con la teología de la liberación, Schickendantz sostiene que "la Teología política surgió, como su pariente latinoamericana, la Teología de la liberación, en el clima cultural de la segunda mitad de los años sesenta; una época compleja, difícil, conflictiva, también extremadamente creativa, incluso para la Iglesia católica" ${ }^{2}$.

Metz se propuso elaborar una teología post-idealista, que superara dos paradigmas teológicos, a saber, el neo-escolástico y el trascendental-idealista, iniciado por su maestro Karl Rahner. Metz sostiene que su propuesta post-idealista busca "en primer lugar, identificar las nuevas crisis y luego, intentar explicar por qué y en qué sentido la teología ahora exigida, que trata estas crisis con intención salvadora, es una teología política"13. Es un paradigma que se sustenta en la crítica y en el lugar destacado que se le concede a la praxis social y política con vistas a una teología del mundo. Ahora bien, Metz tiene la sensación de que ese paradigma solo alcanzará su madurez con la teología de la liberación. Por eso afirma: "y una vez más: quizás es con el proceso de la teología de la liberación cuando llega a ponerse completamente de manifiesto lo que - sobre todo en la vida de la Iglesia- se pretende con este nuevo paradigma"14.

10. R. Gibellini, La teología del siglo XX, o. c., p. 322.

11. J. B. Metz, "El problema de una teología política", o. c., p. 389.

12. C. Schickendantz, "Actualidad y legitimidad de la teología política. Presentación”, en M. Ruz, Nueva teología política, o. c., pp. 15-22, 15. Las cursivas son originales.

13. J. B. Metz, Dios y tiempo. Nueva teología política, p. 121 (Madrid: Trotta, 2002).

14. Ibid., p. 123. 
La cercanía de Metz con América Latina y con la teología de la liberación ha sido múltiple. En primer lugar, recuerda las "distintas visitas a América Latina durante los años ochenta, en las que mis amigos y colegas me permitieron conocer el trabajo comunitario de base donde el trabajo se realizaba desde abajo"15. El impacto de las comunidades eclesiales de base, que surgieron en el continente después del concilio Vaticano II y de la conferencia de Medellín, y la posterior teología de la liberación, impactaron profundamente al teólogo europeo. Los rostros, los relatos y las historias del tercer mundo le permitieron afirmar que "llueve tristeza en Los Andes"16. Esta mística andina y la espiritualidad popular de los creyentes latinoamericanos constituyen un tema recurrente a lo largo de su propuesta teológica.

La experiencia de las comunidades de base se manifiesta en Más allá de la religión burguesa. En esta obra, y particularmente en el capítulo titulado "Cuando cambian los protegidos: en camino a una iglesia de base" ${ }^{17}$, Metz aborda el desafío de la comprensión de la Iglesia como una comunidad creyente mayor de edad, es decir, no solo la autoridad eclesiástica, sino que todos los creyentes son capaces de vivir y de responsabilizarse del evangelio y del cristianismo en medio de la sociedad. A raíz de esto, Metz presenta tres conceptos de Iglesia: la popular tradicional, la del ciudadano burgués y la Iglesia de base, a cada una de ellas le corresponde una teología diferente. La primera es una Iglesia proteccionista, basada en el paradigma neo-escolástico y en los afanes de la apologética. El segundo modelo, el del ciudadano burgués, es la Iglesia de la oferta y del

15. J. B. Metz, "Significado de la teología latinoamericana para mi teología", en L. Susin (ed.), El mar se abrió. Treinta años de teología en América Latina, pp. 138-139, 138 (Santander: Sal Terrae, 2000). Metz recuerda esta visita de la manera siguiente: "En 1988 , invitado por la Goethe Institut, estuve durante cinco semanas viajando y dando conferencias por Latinoamérica, y tuve ocasión de preguntarme una y otra vez qué podría haber de común, aun respetando todas las diferencias entre la nueva teología política de Europa y la teología de la liberación de América Latina. Ambos planteamientos teológicos - intentaba yo responderme entonces y querría repetir aquíse deben en realidad a un temor especial: el temor de que el discurso cristiano sobre Dios en general advierta y atienda demasiado poco la historia lacerante de sufrimiento de los hombres". J. B. Metz, Dios y tiempo, o. c., p. 142. Así, pues, uno de los problemas comunes de estas dos teologías es la teodicea, es decir, qué lugar ocupa Dios en el sufrimiento de los inocentes.

16. J. B. Metz, Dios y tiempo, o. c., p. 238.

17. En este capítulo encontramos referencias a teólogos y a otros teóricos latinoamericanos vinculados con la teología de la liberación, como Leonardo Boff y su eclesiogénesis, Ernesto Cardenal, Fernando Castillo y Paulo Freire. También cita las Conclusiones de Medellín (1968) y Seladoc, centro de documentación teológica de la Facultad de Teología de la Universidad Católica (Chile). Todo ello pone de manifiesto el interés de Metz en el pensamiento teológico latinoamericano y en hacer dialogar su teología política con nuestra teología de la liberación continental. 
servicio, sustentada en la teología liberal-burguesa. Finalmente, está el modelo de la Iglesia de base o de la Iglesia del pueblo, que se basa en las teologías, política y de la liberación, "en una unidad constitutiva de crítica productiva de la Iglesia y de la sociedad"18.

Un tercer momento de la cercanía de Metz con la teología de la liberación es su relación con Gustavo Gutiérrez, quien en su obra emblemática Teología de la liberación. Perspectivas, específicamente en el apartado sobre la dimensión política del evangelio, aborda la nueva teología política. Según Gutiérrez, "la visión escatológica se hace operativa, la teología de la esperanza se hace creadora en contacto con las realidades sociales del mundo de hoy, dando lugar a lo que se ha llamado la teología política" ${ }^{19}$. De esa manera, el teólogo de la liberación remite a los autores que han influido en Metz, entre los que destacan Bloch y Moltmann, sobre todo, por su concepción de la esperanza y de la escatología pensada en clave creadora.

El teólogo peruano es consciente de que "hoy la teología política entra en diálogo fecundo con la teología de la liberación, y se producen interesantes convergencias. Aunque al inicio el acercamiento se hiciera con cierto malestar" ${ }^{\prime 20}$, evidente en las críticas que Gutiérrez hace a la propuesta de Metz.

La primera refiere al lugar desde el cual Metz hace teología, el primer mundo, porque "no le permite calar hondo en la situación de dependencia, de injusticia y de expoliación en que se encuentra la mayor parte de la humanidad" ${ }^{21}$. En segundo lugar, Gutiérrez critica que a su concepto de lo político le falta abordar más la experiencia del conflicto entre los seres humanos. La tercera crítica pide la revisión de algunos de los presupuestos de su teología, con la mediación de las ciencias sociales. Este recurso permitiría elaborar más y mejor la teología política. La utilización del análisis sociológico, especialmente el marxista, fue una tónica de las teologías de la liberación en América Latina, análisis que le supuso una fuerte crítica desde cierto sector de la jerarquía. En esto, Gutiérrez echa de menos un mayor uso de la propuesta de Bloch. Finalmente, Gutiérrez percibe que Metz no se desligó totalmente de la teología de la secularización.

A pesar de sus críticas, Gutiérrez reconoce la importancia teológica de la propuesta de Metz, cuya "nueva teología política se sitúa, con toda claridad, pero de modo original y fecundo en el arco iniciado por la así llamada mayoría de edad de la humanidad"22. Esa aspiración también se expresa en el concepto de una Iglesia popular, que también busca convertirse en una Iglesia adulta y

18. J. B. Metz, Más allá de la religión burguesa, p. 83(Salamanca: Sígueme, 1982).

19. G. Gutiérrez, Teología de la liberación. Perspectivas, p. 332 (Lima: CEP, 2014 ${ }^{13}$ ).

20. G. Gutiérrez, La fuerza histórica de los pobres, p. 236 (Salamanca: Sígueme, 1982).

21. G. Gutiérrez, Teología de la liberación, o. c., p. 339.

22. G. Gutiérrez, La fuerza histórica de los pobres, o. c., p. 236. 
responsable. Asimismo, Gutiérrez reconoce que el proceso vivido en América Latina no es el mismo que el vivido en Europa. Por eso, advierte

... que Metz no tenga en cuenta estas realidades [las de América Latina], se comprende; que no las perciban quienes requieren trasplantar sin más trámites sus ideas, es grave y peligroso [...] la nueva teología política presenta, no obstante, un fecundo esfuerzo por pensar la fe teniendo en cuenta su dimensión política, vale decir tomando conciencia de los problemas más globales y más agudos que vive el ser humano hoy ${ }^{23}$.

Aquí se nos impone una doble advertencia. La primera es leer la teología política en su contexto, considerando las preocupaciones y los temas a los cuales se vio enfrentada y las inquietudes de su autor. Gutiérrez en ello es bastante lúcido, ya que no busca una acomodación simplista de la nueva teología política. Pero a pesar de ello, reconoce que es un planteamiento teológico válido para abordar los temas globales que afectan al ser humano, los cuales, a nuestro juicio, todavía no se han solucionado en la actualidad. Por eso, repensar la teología política sigue teniendo actualidad y vigencia. La segunda es hacer el camino inverso, esto es, la apreciación que Metz hace de la teología de la liberación. Cabe recordar aquí que él prologó una de las ediciones alemanas ${ }^{24}$ de la obra emblemática de Gutiérrez.

En mis prólogos a las anteriores ediciones de Teología de la liberación, de Gustavo Gutiérrez, hice hincapié en algo que merece la pena volver a recordar: el libro - decía yo - vendría a seguir las huellas de la situación histórico-social del propio continente, de la historia social y política de sufrimiento de sus propios pueblos y asumirlas en su misma conciencia teológica ${ }^{25}$.

Lo que está detrás de esta percepción de Metz es la necesidad de elaborar una nueva cultura hermenéutica a partir de los cambios producidos por los movimientos sociales tanto de Europa como de América Latina.

El vínculo entre la teología política europea y la teología de la liberación latinoamericana, según Metz, es la cuestión de la teodicea, es decir, el lugar de Dios en el sufrimiento de los inocentes. Esta cuestión teológica implica, a juicio del teólogo alemán, abandonar tres elementos constitutivos de las teologías posteriores al idealismo. El primero es la inocencia social para recuperar las preguntas centrales de la teología. De esa manera, esta evita caer en el relativismo. Se trata de buscar una teología práctica aliada de la libertad y la justicia. Aquí aparece "el planteamiento de teología fundamental de la nueva teología política, y también de la teología de la liberación en la medida en que es algo más que doctrina

23. G. Gutiérrez, Teología de la liberación, o. c., p. 341.

24. La primera edición alemana data de 1972.

25. J. B. Metz, Dios y tiempo, o. c., p. 141. 
social y pastoral" ${ }^{26}$. En definitiva, se trata de alcanzar una mística política sustentada en la experiencia de las comunidades de base.

El segundo elemento que debe abandonar es la inocencia histórica. "La historia es tema del discurso sobre Dios específicamente cristiano. Y en este sentido la teología tiene que decir adiós a un universalismo histórico sin sujeto, ajeno a toda situación y en cierta medida sin contenido humano"27. Así, pues, Metz recuerda que la teología de la historia es una reflexión fundamental del discurso cristiano sobre Dios. En la recuperación de la historia aparecen los rostros, los relatos, las narraciones, la memoria de los sujetos olvidados. La teología del sujeto se basa en una mística del reconocimiento, que es la mística de Jesús.

No es la mística adusta de ojos cerrados, sino la mística enfática de ojos abiertos [...] y la teología de la liberación, en su búsqueda de sistema, admite la interrupción del rostro de los pobres. Esto la caracteriza, igual que a la teología política, como post-idealista ${ }^{28}$.

Finalmente, el último elemento que debe superarse es la supuesta inocencia étnico-cultural. Metz plantea una teología que aborde el desafío de la construcción de una Iglesia policéntrica y multicultural, para lo cual es necesario construir una cultura hermenéutica basada en el reconocimiento de los otros, de la alteridad, del respeto y del encuentro. En efecto, "en el central mandamiento bíblico del amor al prójimo, este prójimo no lo forman principalmente los cercanos, sino los distintos, los otros extraños. Y en este sentido el logos de la teología tiene que dejarse interrumpir por el rostro de estos otros extraños" ${ }^{29}$. Los sujetos de la teología y la teología del sujeto han de fundamentarse en una mentalidad del reconocimiento y de la eclesialidad. Esta es una fe no reducida a una experiencia individualista y, por lo tanto, la consideración de una mística política y social cercana a la historia, a una fe eminentemente comunitaria.

\section{Teologías de la praxis}

La teología política y la teología latinoamericana de la liberación se encuentran dentro de las llamadas teologías de la praxis, cuya intención era reflexionar en torno al comportamiento concreto de la persona y de los grupos humanos. Buscaron una orientación más concreta que especulativa, más científica que metafísica, para alcanzar su objetivo principal: "presentar el fermento práctico, concreto, social, político e interpersonal de la fe" ${ }^{30}$. Así nacieron teologías de la cultura, del mundo, del trabajo, de la paz, política o de la liberación. Estas "teologías

26. Ibid., p. 143 .

27. Ibid., p. 144.

28. Ibidem.

29. Ibid., p. 145.

30. B. Mondin, Teologías de la praxis, p. 5 (Madrid: BAC, 1981). 
del genitivo", como también son denominadas, provienen de movimientos eclesiales previos. Otras fueron impulsadas por Gaudium et spes, que buscó constituir una teología del mundo y del encuentro y del diálogo. De ahí que se pueda afirmar que Gaudium et spes, una Constitución pastoral, constituye tanto un punto de llegada como un punto de partida para las teologías de la praxis.

Se llama constitución pastoral porque, apoyada en principios doctrinales, quiere expresar la actitud de la Iglesia ante el mundo y el hombre contemporáneo. Por ello, ni en la primera parte falta intención pastoral, ni en la segunda intención doctrinal (nota 1).

De esta manera, la Iglesia del concilio renueva la forma de expresar su interés por encontrarse con las culturas y la modernidad, un encuentro que busca hacerse significativo y concreto sin abandonar los principios de la fe cristiana. C. Casale sostiene que la dimensión pastoral aparece como un género literario, un lenguaje propio e inédito del Vaticano II y un paso original y creativo, ya que "el aspecto pastoral se ha convertido en el criterio primordial utilizado en la formulación y en la presentación de la verdad y no simplemente en el motivo para adoptar decisiones prácticas" ${ }^{31}$. Ahora bien, la Iglesia no se agota en lo pastoral, ni en las decisiones prácticas, sino que ellas constituyen un vehículo imprescindible para hacer llegar el depositum fidei a los nuevos oyentes del evangelio y de las enseñanzas de la fe.

La recepción del Vaticano II, en general, y de Gaudium et spes, en particular, se realiza de manera creativa en las teologías de la praxis, sobre todo, en la teología de la liberación latinoamericana y en la teología política europea, que asumieron el llamado primado de la praxis, o el paso de la ortodoxia a la ortopraxis. De esa manera, la teología la consideran como un momento segundo, que reflexiona sobre la praxis que se realizó primero. Es a propósito de esto que G. Gutiérrez define la teología como una "reflexión crítica sobre la praxis"32.

En Teología de la liberación, Gutiérrez expresa que la praxis se puede evidenciar en tres momentos de la vida cristiana. El primero es el primado del amor, entendido como toma de postura ante la vida y como entrega al otro y a los otros. El segundo es la espiritualidad que se hace liberadora y contemplativa en la acción. Este concepto de espiritualidad liberadora también tendrá eco en la teología de Metz, cuando habla de la espiritualidad y la mística, que deja de ser trascendental para constituirse en mística política.

El tercer momento es considerar la vida de la Iglesia como lugar teológico en cuanto que "la Palabra de Dios convoca, y se encarna en la comunidad de

31. C. Casale, "El género literario pastoral del Concilio Vaticano II como clave interpretativa de su identidad y relevancia", Palabra y Razón IV (2013), 7-25, 13.

32. G. Gutiérrez, Teología de la liberación, o. c., p. 72. 
fe que se entrega al servicio de la humanidad" ${ }^{33}$. Un servicio que se realiza mediante el discernimiento de los signos de los tiempo de cara a la transformación del mundo y a la escatología, que permite concebir la historia humana abierta al futuro, al encuentro definitivo del ser humano con sus hermanos y del género humano con Dios.

En síntesis, la teología de la liberación y la teología europea afirman que la praxis es acción transformadora, liberadora y crítica. La praxis puede renovar y transforma la naturaleza y la realidad actual, tal como lo propuso la filosofía marxista. Así, pues, la praxis no es una simple repetición inconsciente, sino que debe configurarse como una acción creadora, consciente y radical al enfrentar la realidad que pretende cambiar. En cuanto acción creadora, la praxis es emancipadora, porque cuando se sitúa ante determinada realidad, tiene la pretensión de transformarla y de convertirla en algo mejor.

Ahora bien, la propuesta de la praxis no se agota en los actos, sino que también reclama una acción reflexiva, es decir, una praxis teórica, que sabe hacia dónde se dirige su práctica, que sabe lo que hace y que busca discernir la acción y cómo conseguir los objetivos propuestos por la persona y por los grupos humanos. Desde la vivencia de la fe y de la teología, la praxis busca acoger la revelación en la historia, el lugar teológico de la acción de Dios, para transformar esa misma historia, ya que asume el desafío de humanizarla. Por eso, "el mayor desafío hoy consiste en desarrollar una praxis cristiana creativa y pascual; una que pueda ser al mismo tiempo cultural y contracultural [...] hay cristianismo allí donde la praxis cristiana se ejerce en favor de todos a partir de los últimos" ${ }^{\prime 34}$.

Solo así se puede seguir verdaderamente a Cristo Jesús, un seguimiento necesariamente eclesial.

\section{Metz y las situaciones de pecado social}

La teología política de Metz es una teología fundamental. En cuanto fundamento de la fe cristiana, parte necesariamente de la revelación de Dios y de la fe en el ser humano, entendida como respuesta a la manifestación divina. Así, en relación a la respuesta creyente, Metz afirma lo siguiente: "La fe de los cristianos es una praxis dentro de la historia y de la sociedad que se concibe como esperanza solidaria con el Dios de Jesús, en cuanto Dios de vivos y muertos que llama a todos a ser sujetos en su presencia" ${ }^{35}$.

33. Ibid., p. 77 .

34. J. Costadoat, "Discernimiento de la praxis cristiana en la cultura moderna", Palabra y Razón IV (2013), 115-125, 121-122.

35. J. B. Metz, La fe en la historia y la sociedad, p. 91 (Madrid: Cristiandad, 1979). 
La praxis, según Metz, está relacionada con el seguimiento de Jesús, cuyo fundamento consiste en la lucha histórica por el ser humano de tal manera que todos lleguen a vivir una solidaridad universal.

El pensamiento de Metz se articula en el concepto fundamental de memoria, expresado en dos momentos: la memoria pasionis y la memoria resurrectionis. Estos conceptos fundamentan el enunciado teológico básico de la teología política, a saber, preservar la memoria peligrosa del Dios mesiánico, del Dios de la resurrección de los muertos y del Dios del juicio. La "memoria peligrosa" coloca en el centro de la reflexión teológico-política "el sufrimiento de las víctimas del pasado, para atender sus padecimientos y aceptar el desafío de los muertos" ${ }^{36}$.

El pecado social lleva en sí la marca del sufrimiento de las víctimas, un reflejo del Dios mesiánico crucificado. Metz sostiene que frente a la situación de sufrimiento y de una historia de los vencidos, el mensaje cristiano de redención se ofrece como mensaje liberador y dador de sentido. Esta historia de salvación concreta la "libertad nacida de la liberación redentora de Dios en la cruz de Jesús"37.

En la realidad marcada por el pecado, tanto personal como social, la política tiene una necesaria vinculación con la moral. Según Metz, esa relación "exige la movilización de las fuerzas espirituales y morales mediante una radical democratización de la infraestructura de la sociedad estableciendo la libertad y la responsabilidad desde abajo" 38 .

El lugar desde el cual el cristiano se compromete a seguir a Jesús es la crítica de las estructuras del sufrimiento y la transformación de dichas situaciones a la luz de la redención obrada por Jesucristo al vencer a la muerte con su resurrección.

Así, pues, la historia del sufrimiento del mundo no queda sujeta a la muerte de la cruz, sino que asume la esperanza en la memoria resurrectionis. La resurrección de Jesucristo posee en sí una connotación política, porque "insiste en la memoria del sufrimiento acumulado en la historia y desde allí determina nuestras acciones y esperanza" ${ }^{39}$. Gracias a la resurrección, los que han sufrido las consecuencias del pecado social vuelven a tener voz y tienen una palabra que decir. La justicia se asume entonces como la respuesta de la revelación y de la gracia frente a la situación del pecado.

36. M. Ruz, G. Rosolino y C. Schickendantz, "La fuerza subversiva del sufrimiento evocado. Recepción de Walter Benjamin en la teología de Johann Baptist Metz", Teología XLVI (2009), 397-420, 413.

37. J. B. Metz, La fe en la historia, o. c., p. 141.

38. Ibid., p. 114.

39. M. Ruz, G. Rosolino y C. Schickendantz, "La fuerza subversiva del sufrimiento evocado", o. c., p. 413. 


\section{Conclusiones}

El encuentro de Metz, teólogo del primer mundo, con la realidad de dolor, de pobreza y de marginación, pero, sobre todo, de fe del tercer mundo significa una recepción positiva de esta nueva forma de hacer teología. Es más, el mismo Metz reconoce la importancia de la teología de la liberación en su propia teología. Aquí, en el encuentro y la acogida de estas teologías, es donde se inscribe el interés de este artículo.

La teología política constituye un aporte fundamental para pensar el sufrimiento de la historia humana por su toma de conciencia de la responsabilidad histórica de la Iglesia y de la sociedad respecto al porvenir de la humanidad y a la transformación de la sociedad. El rescate de la historia de los vencidos contribuye a asumir la defensa de la dignidad de la persona humana de una manera nueva y decidida. Asimismo, el sentido de la responsabilidad histórica nos coloca ante la exigencia de constituirnos en comunidades creyentes y sociopolíticas compasivas, para lo cual es necesario asumir la historia, pero no de manera aislada, sino preocupándonos los unos por los otros, sobre todo, por los que más sufren.

Por eso, la teología política se presenta como instancia legítima y necesaria para proponer una nueva reforma eclesial en la cual, desde la identidad comunitaria, podamos reconocernos y reconocer a la sociedad política y religiosamente pluralista, en la que nos encontramos. En definitiva, la teología política, al igual que la teología de la liberación, busca ser teología del mundo, reflexiones creyentes que, al hacerse praxis, anuncien la experiencia cristiana de Dios desde el sufrimiento de la historia humana. Un Dios que quiere dialogar con la cultura contemporánea desde la mística política y la oración, desde el reconocimiento de la injusticia actual y desde el esfuerzo para liberar de las esclavitudes sociales, económicas, políticas, educativas y religiosas. 